\title{
Evaluation the Recurrence Risk of Gestational Trophoblastic Neoplasia (GTN) after Serum $\beta$ hCG Normalization
}

\author{
Azar Ahmadzadeh ${ }^{1}$, Elham Karimi Moghaddam ${ }^{1}$ \& Elahe Shafieyan ${ }^{1}$ \\ ${ }^{1}$ Department of Obstetrics and Gynecology, Ahvaz Jondishapour University of Medical Sciences, Ahvaz, Iran \\ Correspondence: Elahe Shafieyan, Department of Obstetrics and Gynecology, Ahvaz Jondishapour University of \\ Medical Sciences, Ahvaz, Iran.
}

Received: March 29, 2020

Accepted: May 19, $2020 \quad$ Online Published: November 30, 2020

doi:10.5539/jmbr.v10n1p194

URL: https://doi.org/10.5539/jmbr.v10n1p194

\begin{abstract}
Background: Although many GTN patients can be treated with chemotherapy, a small proportion of them will relapse after complete recovery. To the best of our knowledge, there is not any information in respect of relapsed GTN cases in our region. In the current study we have aimed to evaluation of the recurrence risk of gestational trophoblastic neoplasia (GTN) after serum $\beta$ hCG normalization

Methods: This descriptive-analytical study was carried out on registered hospital data of patients with confirmed GTN diagnosis following molar pregnancy who admitted to the gynecology ward of Imam Khomeini Hospital between 2011 and 2017. Patients with diagnosis of postmolar GTN, based on at least five bhcg measurements was included. Patients information including initial level of serum BhcG, time to Bhcg resolution, types of molar pregnancy, treatment protocols, need to recuretage relapse, and finally, the period time between bhcg resolution to relapse were evaluated.

Results: In the present study, 239 patients with GTN (including 180 complete and 59 partial moles) were evaluated. The mean age of the patients was 28.8 years, ranging from 16 to 47 years. The mean $\beta \mathrm{hCG}$ concentration was $170,000 \mathrm{IU} / \mathrm{ml}$ (ranged 760 to 850,000 ). The mean time of $\beta \mathrm{hCG}$ resolution was 8.19 months in the range of 4 to 12 months. Recurrence was observed in 9 patients (3.7\%). The mean period time between $\beta \mathrm{hCG}$ resolution to relapse was 20.94 months. The mean initial level of $\beta \mathrm{hCG}$ was significantly lower in patients with recurrence $(\mathrm{p}<0.0001)$. The highest recurrence rate was seen in those receiving multiple-drug chemotherapy. There was also a significant relationship between disease stage and recurrence rate.

Conclusion: The findings of this study indicate that although the recurrence of GTN is relatively low, given the poor prognosis of these patients, continuous evaluation of bHCG levels for at least two years is essential to prevent disease progression.
\end{abstract}

Keywords: Gestational Trophoblastic Neoplasia, Molar Pregnancy, Recurrence

\section{Introduction}

Gestational trophoblastic disease (GTD) refers to a group of diseases characterized by abnormal trophoblastic tissue proliferation. Gestational trophoblastic neoplasia (GTN), a malignant form of GTD, includes invasive mole, choriocarcinoma, placental trophoblastic tumor, and epithelioid trophoblastic tumor. Although these malignancies occur weeks or years after all types of pregnancy, they are most commonly followed by molar pregnancies (Heller, 2015; Barroilhet, 2018; Shaaban, Rezvani, Haroun, Kennedy, Elsayes, Olpin, Salama, Foster, \& Menias, 2017; Reva Tripathi, 2017; Biscaro, Braga, \& Berkowitz, 2015). According to the International Federation of Obstetrics and Gynecology (FIGO) recommendation, GTN is diagnosed by observing each of the four criteria: no decrease in hCG- $\beta$ levels after four weeks, elevated hCG- $\beta$ serum level for three consecutive weeks, hCG- $\beta$ detection 9-month after mole removal and histological diagnosis of choriocarcinoma, (Eysbouts, Ottevanger, Massuger, IntHout, Short, Harvey, Kaur, Sebire, Sarwar, Sweep, \& Seckl, 2017). Some of the patients with molar pregnancies did not completely cure after mole removal and developing to malignancy. Therefore, finding an appropriate marker for the early prediction of neoplasia has been critically important (Seckl, Sebire, \& Berkowitz, 2010). Women with GTN usually characterized by vaginal bleeding in the first trimester or abnormal ultrasound at 12 to 18 weeks of gestation. In some cases, invasion of the tumor into the uterus leads to significant vaginal bleeding, and in other cases, it can lead to intraperitoneal hemorrhage 
by perforating the myometrium. Besides, an intrauterine necrotizing tumor may act as a foci of infection (Mangili, Garavaglia, Cavoretto, Gentile, Scarfone, \& Rabaiotti, 2008).

Numerous studies have been conducted in recent years to find the appropriate markers for the early prediction of GTN. For example, a series of studies suggested the ratio of B-HCG before and a week after mole removal or the ratio of hCG- $\alpha$ and hCG- $\beta$ as appropriate early predictors of GTN (Kang, Choi, \& Kim, 2012). GTD is considered treated if the hCG measurement is normal for three consecutive weeks (less than five $\mathrm{mIU} / \mathrm{mL}$ ) and then stays normal for up to 9 months. However, the main problem in developing countries is the stopping of follow up after hCG normalization, and only half of the patients attend scheduled medical visits after molar pregnancy (Schmitt, Doret, Massardier, Hajri, Schott, Raudrant, \& Golfier, 2013).

The reports of relapsed patients indicated the possibility of GTN recurrence after hCG normalization. Bagshawe et al. Showed that GTN could recur automatically after normalization of serum hCG levels. Therefore, a common follow-up protocol is used after normalizing hCG levels. Recently published guidelines suggested the monitoring of hCG levels at least nine months after its normalization (Jankilevich, Uberti, Braga, Bianconi, Maesta, Viggiano, Sun, Cortes Charry, Salazar, Grillo, \& Moreira de Andrade, n. d.). In the current study, we have aimed to investigate the incidence of relapsed GTN after normalization of serum hCG levels.

\section{Material and Methods}

\subsection{Study Design}

This descriptive-analytical study registered hospital data of patients with confirmed GTN diagnosis following molar pregnancy who admitted to the gynecology ward of Imam Khomeini Hospital between 2011 and 2017. Patients with diagnosis of postmolar GTN, based on at least five bhcg measurements was included, while those with incomplete records were excluded. Patients information such as initial level of serum BhcG, time to Bhcg resolution, types of molar pregnancy, treatment protocols, need to recuretage relapse, and finally, the period time between bhcg resolution to relapse were evaluated during the study years.

\subsection{Definitions}

Postmolar GTN was defined as having one of the following FIGO criteria: When the plateau of hCG lasts for four measurements over a period of 3 weeks or longer; that is, days 1, 7, 14, 21.

When there is a rise in hCG for three consecutive weekly measurements over at least a period of 2 weeks or more; days $1,7,14$.

If there is a histologic diagnosis of choriocarcinoma.

\subsection{Statistical Analysis}

The data were described by mean, median, standard deviation, frequency, and percentage. The mean comparison was carried out by independent student t-test or Mann-Whitney. Proportions were compared by the chi-square test. Kaplan Meier plot was used to describe the relapse time. All statistical analysis was done by SPSS version 20.

\section{Results}

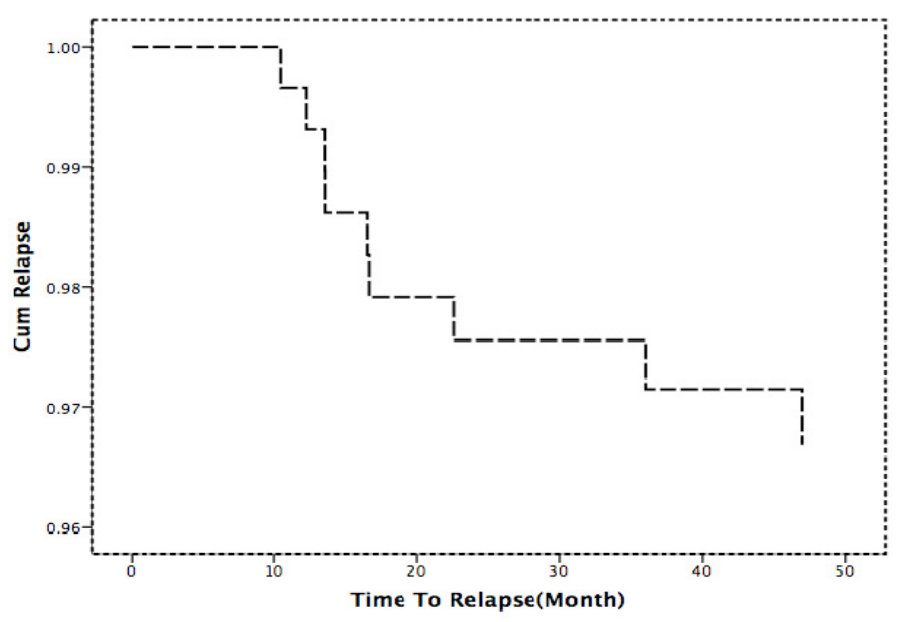

Figure 1. The time of $\beta \mathrm{hCG}$ resolution to relapse 
Table 1. Descriptive analysis of the patients

\begin{tabular}{ll}
\hline Characteristics & Mean(range) \pm SD - Frequency(\%) \\
\hline Age & $28.8(16-47) \pm 6.2$ \\
bHCG & $170102(760-850000) \pm 281000$ \\
Pregnancy Age & $7.8(5-11) \pm 1.22$ \\
Time to Resolution (month) & $8.19(4-12) \pm 2.44$ \\
GTN Type & \\
Complete Mole & $180(75.3 \%)$ \\
Partial Mole & $59(24.7 \%)$ \\
Treatment & \\
Re-Curettage & $16(6.7 \%)$ \\
Single drug Chemotherapy & $157(65.7 \%)$ \\
Multiple drug Chemotherapy & $66(27.6 \%)$ \\
Relapse & \\
Yes & $9(3.7 \%)$ \\
No & $232(96.3 \%)$ \\
Mean time to relapse & 20.94 \\
\hline
\end{tabular}

In the present study, 239 patients with GTN (including 180 complete and 59 partial moles) were evaluated. The mean age of the patients was 28.8 years, ranging from 16 to 47 years. The mean $\beta \mathrm{hCG}$ concentration was $170,000 \mathrm{IU} / \mathrm{ml}$ (ranged 760 to 850,000 ). The mean time of $\beta$ hCG resolution was 8.19 months in the range of 4 to 12 months. Most patients (157 patients) were treated with single-drug chemotherapy, including MTX (26 patients) and actinomycin (131 patients). Also, 66 patients (27.6\%) were treated with multi-drug chemotherapy, and 16 patients $(6.7 \%)$ were treated with recurettage. Recurrence was observed in 9 patients $(3.7 \%)$. The mean period time between $\beta \mathrm{hCG}$ resolution to relapse was 20.94 months (Table 1) (Figure 1).

Our patients were divided into two groups, with recurrence and with out recurrence The Mean age of patients in both groups was not statistically significant. The mean initial level of $\beta \mathrm{hCG}$ was significantly lower in patients with recurrence $(\mathrm{p}<0.0001)$. There was no significant difference in gestational age between the two groups. Also, the mean period time of $\beta \mathrm{hCG}$ normalization was not significantly different in both groups $(0.66)$. The highest recurrence rate was seen in those receiving multiple-drug chemotherapy. There was also a significant relationship between disease stage and recurrence rate (Table 2). In relapsed group two patients were in stage 1, two in stage 2 , three in stage 3 and two in stage 4.

Table 2. Comparison of studied variables in patients with and without recurrence

\begin{tabular}{llll}
\hline \multirow{2}{*}{ Characteristics } & \multicolumn{1}{c}{ Relapsed GTN Vs non-Relapsed } & P value \\
\hline Age & Relapsed & Non-Relapsed & 0.445 \\
bHCG & $27.33 \pm 6.8$ & $28.9 \pm 6.2$ & $\mathrm{p}<0.0001$ \\
Pregnancy Age & 42847 & 175039 & 0.11 \\
Time to Resolution (month) & $8.51 \pm 1.12$ & $7.8 \pm 1.22$ & 0.66 \\
Treatment & $8.44 \pm 2.18$ & $8.09 \pm 2.4$ & $\mathrm{p}<0.0001$ \\
Re-curettage & & & \\
Single drug Chemotherapy & $1(6.3 \%)$ & $15(93.8 \%)$ & $\mathrm{p}<0.0001$ \\
Multiple drug Chemotherapy & $3(1.9 \%)$ & $154(98.1 \%)$ & \\
Stage & $5(7.6 \%)$ & $61(92.4 \%)$ & \\
I & & & \\
II & $2(0.9 \%)$ & $228(99.1 \%)$ & \\
III & $2(66.7 \%)$ & $1(33.3 \%)$ & \\
IV & $3(60 \%)$ & $2(40 \%)$ & \\
\hline
\end{tabular}

The mean period time between $\beta$ hCG resolution to relapse was evaluated in different treatment modalities. Accordingly, patients treated with multi-drug chemotherapy showed the shortest treatment time to relapse (Table 3) (Figure 2). 


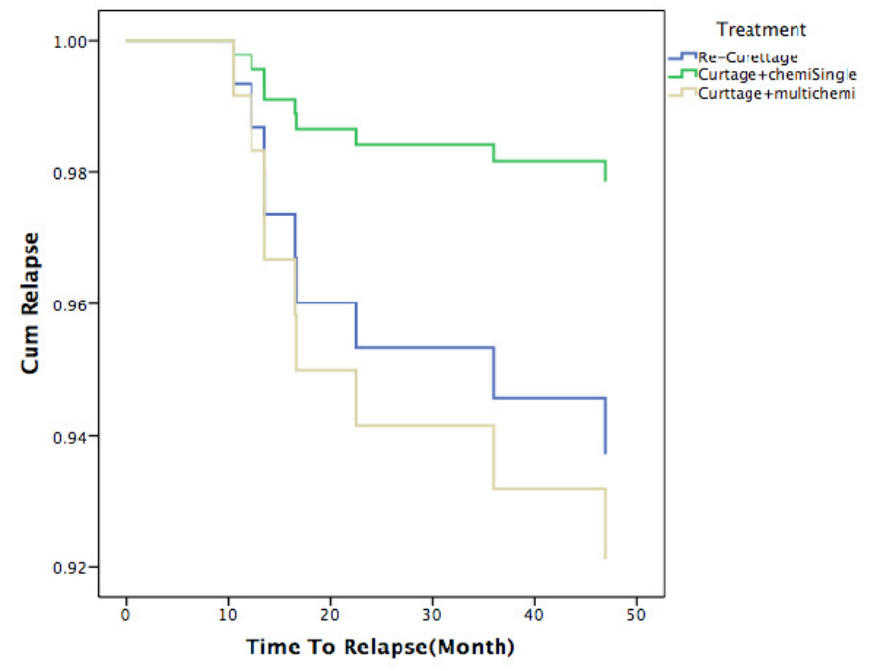

Figure 2. The time of $\beta \mathrm{hCG}$ resolution to relapse in different treatment modalities

Table 3. The mean period time between $\beta$ hCG resolution to relapse in different treatment modalities

\begin{tabular}{lllllll}
\hline Treatments & $\mathrm{N}$ & Mean & Std. Deviation & Std. Error & Minimum & Maximum \\
\hline Re-Curettage & 1 & 47.00 & $\cdot$ &. & 47 & 47 \\
ChemiSingle & 3 & 20.9 & 13.3 & 7.69 & 10 & 36 \\
Multichemi & 5 & 15.7 & 4.188 & 1.710 & 12 & 23 \\
Total & 9 & 20.94 & 12.45 & 4.15 & 10 & 47 \\
\hline
\end{tabular}

There were three relapses in patients treated with single-drug chemotherapy, one in MTX and two in actinomycin. The recurrence rate ratio was not significantly different between the patients treated with MTX or Actinomycin. Of the 66 patients treated with multi-drug chemotherapy, 20 patients were high risk and initially treated with multi-drug therapy, while other 46 patients were secondary treated with this protocol due to non-response to single-drug chemotherapy (Table 4).

Table 4. Comparison of treatment protocol in patients with and without recurrence

\begin{tabular}{|c|c|c|c|}
\hline \multirow{2}{*}{ Characteristics } & \multicolumn{2}{|c|}{ Relapsed GTN Vs non-Relapsed } & \multirow{2}{*}{$P$ value } \\
\hline & Relapsed & Non-Relapsed & \\
\hline \multicolumn{4}{|l|}{ Single drug Chemotherapy } \\
\hline MTX & $1(3.8 \%)$ & $25(96.2 \%)$ & \multirow{2}{*}{0.421} \\
\hline Actinomycin & $2(1.5 \%)$ & $129(98.47 \%)$ & \\
\hline Mean chemotherapy courses & 10.6 & 11.14 & 0.281 \\
\hline \multicolumn{4}{|l|}{ Mutidrug Chemotherapy } \\
\hline High risk patients & $2(10 \%)$ & $18(90 \%)$ & \multirow{2}{*}{0.63} \\
\hline Not response to Single drug chemotherapy & $3(6.52 \%)$ & $43(93.47 \%)$ & \\
\hline Mean chemotherapy courses & $7.3 \pm 1.5$ & $6.3 \pm 1.2$ & 0.199 \\
\hline
\end{tabular}

\section{Discussion}

Although many GTN patients can be treated with chemotherapy, a small proportion of them will relapse after complete recovery. Previous studies have carried out on a low sample size due to its low frequency. To the best of our knowledge, there is not any information in respect of relapsed GTN cases in our region. Therefore, this retrospective cohort study compared the patients with and without relapsed GTN.

The recurrence rate in the present study was $3.7 \%$. It was similar to previous studies. Yang et al. In a study of 1130, patients have reported the 314 patients with recurrent GTN (3.4\%), which was similar to the results 
obtained in this study (Yang, Xiang, Wan, \& Yang, 2006). However, another study by the same group found a $6.5 \%$ recurrence GTN rate between, which was much more than our study findings (Kong, Zong, Cheng, Jiang, Wan, Feng, Ren, Zhao, Yang, \& Xiang, 2020). Also, Barga et al., In a study on GTN, followed molar pregnancy, showed that 10 of 2284 GTN patients would relapse (Braga, Maestá, Matos, Elias, Rizzo, \& Viggiano, 2015).

Moreover, our results showed that the meantime of $\beta \mathrm{hCG}$ resolution to relapse was 20.1 months. The lowest recurrence time was ten months, and some patients showed recurrence even after 47 months. Consistent with the present study in a study by Barga et al., The mean period time of diagnosis to recurrence was 18 months, and all of the diagnoses were made 9 months after $\beta$ hCG resolution (Braga, Maestá, Matos, Elias, Rizzo, \& Viggiano, 2015). However, in the study of Yang and his colleagues, this time was three months, which was below our results. In the Yang study, in contrast to the present study, the median duration of recurrence was reported, whereas, in our study, the meantime was reported. Besides, similar to the present study in the Yang study, it was shown that more than $78 \%$ of patients had recurrence within one year after treatment, and $10 \%$ had recurrence after two years (Kong, Zong, Cheng, Jiang, Wan, Feng, Ren, Zhao, Yang, \& Xiang, 2020). These findings indicate the need for long term monitoring of bHcg levels after complete recovery. Balchandran and colleagues in a study on 4,000 GTN patients have suggested that the level of Bhcg should be monitored for at least one year after complete recovery (Balachandran et al., 2019).

Our findings also showed that initial B-hcg levels were significantly lower in patients with recurrence. These findings were in line with the study by Powles et al. However, the cause is not well understood. But the tumor in these patients would likely consist of mutated cells and cells with low maturation without the ability to produce Bhcg. This condition has been shown in other tumors (Fosså, Waehre, \& Paus, 1992). Besides, the type of treatment was also related to the incidence of recurrence. Patients undergoing multiple drug chemotherapy have been shown to have a higher incidence of GTN recurrence.

Given that high-risk patients are being treated with multi-drug chemotherapy regimens, the higher incidence of recurrence in these patients may have been due to high-risk GTN. A study by Yang and colleagues also showed that the recurrence rate in high-risk patients was $6.9 \%$ and approximately four times higher than the low-risk patients (Kong, Zong, Cheng, Jiang, Wan, Feng, Ren, Zhao, Yang, \& Xiang, 2020). Couder and colleagues also found that patients who required more than four doses of MTX to normalize bhCG levels had a significantly higher recurrence risk (Couder, Massardier, You, Abbas, Hajri, Lotz, Schott, \& Golfier, 2016).

\section{Conclusion}

The findings of this study indicate that although the recurrence of GTN is relatively low, given the poor prognosis of these patients, continuous evaluation of bHCG levels for at least two years is essential to prevent disease progression. In the current study, we have evaluated the GTN patients of Khuzestan for the first time, and it was the strength of our study. We did not study the patietns survival rate, and it was the limitation of the study.

\section{Conflict of interests}

The authors declare that there is no conflict of interests regarding the publication of this paper.

\section{References}

Balachandran et al. (2019). When to stop human chorionic gonadotrophin (hCG) surveillance after treatment with chemotherapy for gestational trophoblastic neoplasia (GTN): A national analysis on over 4,000 patients. Gynecol Oncol, 155, 8-12.

Barroilhet, L. M. (2018). Gestational Trophoblastic Disease. Gynecologic Care, 360.

Biscaro, A., Braga, A., \& Berkowitz, R. S. (2015). Diagnosis, classification and treatment of gestational trophoblastic neoplasia. Revista Brasileira de Ginecologia e Obstetrícia, 37(1), $42-51$.

Bolze, P. A., Riedl, C., Massardier, J., Lotz, J. P., You, B., Schott, A. M., Hajri, T., \& Golfier, F. (2016). Mortality rate of gestational trophoblastic neoplasia with a FIGO score of $\geqslant 13$. American Journal of Obstetrics and Gynecology, 214(3), 390-e1.28.

Braga, A., Maestá, I., Matos, M., Elias, K. M., Rizzo, J., \& Viggiano, M. G. (2015). Gestational trophoblastic neoplasia after spontaneous human chorionic gonadotropin normalization following molar pregnancy evacuation. Gynecologic Oncology, 139(2), 283-7.

Couder, F., Massardier, J., You, B., Abbas, F., Hajri, T., Lotz, J. P., Schott, A. M., \& Golfier, F. (2016). Predictive factors of relapse in low-risk gestational trophoblastic neoplasia patients successfully treated with methotrexate alone. American Journal of Obstetrics and Gynecology, 215(1), 80-e1. 
Eysbouts, Y. K., Ottevanger, P. B., Massuger, L. F., IntHout, J., Short, D., Harvey, R., Kaur, B., Sebire, N. J., Sarwar, N., Sweep, F. C., \& Seckl, M. J. (2017). Can the FIGO 2000 scoring system for gestational trophoblastic neoplasia (GTN) be simplified A new retrospective analysis from a nationwide data-set. Annals of Oncology.

Fosså, S. D., Waehre, H., \& Paus, E. (1992). The prognostic significance of prostate specific antigen in metastatic hormone-resistant prostate cancer. British Journal of Cancer, 66(1), 181-4.

Heller, D. S. (2015). Gestational Trophoblastic Neoplasia. In OB-GYN Pathology for the Clinician 2015 (pp. 215-227). Springer International Publishing.

Jankilevich, G., Uberti, E., Braga, A., Bianconi, M. I., Maesta, I., Viggiano, M., Sun, S., Cortes Charry, R., Salazar, A., Grillo, B. M., \& Moreira de Andrade, J. (n. d.). Treatment of patients with gestational trophoblastic neoplasia (GTN) in 12 South America referral centers: Results after 10 years since international FIGO consensus.

Kang, W. D., Choi, H. S., \& Kim, S. M. (2012). Prediction of persistent gestational trophobalstic neoplasia: The role of hCG level and ratio in 2weeks after evacuation of complete mole. Gynecologic Oncology, 124(2), 250-3.

Kong, Y., Zong, L., Cheng, H., Jiang, F., Wan, X., Feng, F., Ren, T., Zhao, J., Yang, J., \& Xiang, Y. (2020). Management and risk factors of recurrent gestational trophoblastic neoplasia: An update from 2004 to 2017. Cancer Medicine.

Mangili, G., Garavaglia, E., Cavoretto, P., Gentile, C., Scarfone, G., \& Rabaiotti, E. (2008). Clinical presentation of hydatidiform mole in northern Italy: has it changed in the last 20 years. American Journal of Obstetrics and Gynecology, 198(3), 302-e1.

Reva Tripathi, M. S. (2017). Gestational Trophoblastic Neoplasia. Evidence Based Clinical Gynecology.

Schmitt, C., Doret, M., Massardier, J., Hajri, T., Schott, A. M., Raudrant, D., \& Golfier, F. (2013). Risk of gestational trophoblastic neoplasia after hCG normalisation according to hydatidiform mole type. Gynecologic Oncology, 130(1), 86-9.

Seckl, M. J., Sebire, N. J., \& Berkowitz, R. S. (2010). Gestational trophoblastic disease. The Lancet, 376(9742), 717-29..

Shaaban, A. M., Rezvani, M., Haroun, R. R., Kennedy, A. M., Elsayes, K. M., Olpin, J. D., Salama, M. E., Foster, B. R., \& Menias, C. O. (2017). Gestational Trophoblastic Disease: Clinical and Imaging Features. Radio Graphics, 37(2), 681-700.

Yang, J., Xiang, Y., Wan, X., \& Yang, X. (2006). Recurrent gestational trophoblastic tumor: Management and risk factors for recurrence. Gynecologic Oncology, 103(2), 587-90.

\section{Copyrights}

Copyright for this article is retained by the author(s), with first publication rights granted to the journal.

This is an open-access article distributed under the terms and conditions of the Creative Commons Attribution license (http://creativecommons.org/licenses/by/4.0/). 